Europhysics Letters

PREPRINT

\title{
Hard colloidal rods near a soft wall: wetting, drying, and symmetry breaking
}

\author{
Kostya Shundyak ${ }^{1}$ and René van RoiJ ${ }^{2}$ \\ 1 Instituut-Lorentz for Theoretical Physics, Leiden University - Niels Bohrweg 2, Lei- \\ den, NL-2333 CA, The Netherlands. \\ 2 Institute for Theoretical Physics, Utrecht University - Leuvenlaan 4, 3584 CE Utrecht, \\ The Netherlands.
}

PACS. 61.30.Hn - Surface phenomena: surface-induced ordering, wetting.

PACS. 64.70.Md - Transitions in liquid crystals.

PACS. 05.70.Np - Interface and surface thermodynamics.

\begin{abstract}
Within an Onsager-like density functional theory we explore the thermodynamic and structural properties of an isotropic and nematic fluid of hard needle-like colloids in contact with a hard substrate coated with a soft short-ranged attractive or repulsive layer. As a function of the range and the strength of the soft interactions we find wetting and drying transitions, a pre-drying line, and a symmetry-breaking transition from uniaxial to biaxial in the wetting and drying film.
\end{abstract}

Whereas bulk liquid crystal phases of suspensions of colloidal hard rods have been essentially understood due to Onsager's work in the 1940's [1,2] and simulations [3] and density functional theories [4-6] in the late 1980's, their surface and interfacial properties are subject of ongoing study, not only experimentally but also theoretically and numerically. Good progress was made during the past decade in the theoretical study of planar free isotropic-nematic (IN) interfaces, e.g. it is known by now that the nematic director in the thermodynamically stable state is parallel to the interfacial plane [7-9], that complete wetting of the IN interface by another nematic phase occurs near the triple point in binary rod mixtures [10], and that the one-particle distributions in the IN interface are only weakly biaxial [11-13]. The effect of external substrates on suspensions of hard rods has also been studied. For a planar hard wall, for instance, evidence of complete wetting of the wall-isotropic (WI) interface by an intervening nematic film was provided by theory [14] and simulations [15]. Other studies were concerned with properties of a hard-rod fluid in contact with a "penetrable" wall, which restricts only the translational degrees of freedom of the rods [16]. It was shown that such a wall favors homeotropic anchoring of the nematic director, and that the WI interface exhibits complete wetting by the homeotropically aligned nematic phase upon approach of IN coexistence $[8,17]$. The common feature of all these studies is that the chosen wall potential does not allow to control the degree of surface nematic order. This is in contrast with Landau-de Gennes theory, which predict rich surface phase diagrams for liquid crystals [18-20]. A drawback of this formalism is, however, that the effects of particular surface-particle interactions are hidden in

(C) EDP Sciences 
expansion coefficients that are not always easily traced back to the microscopic details [20,21]. Therefore, it is interesting and relevant to construct surface phase diagrams, for a "simple" fluid of monodisperse hard rods, in terms of parameters of the surface potential.

Calculating surface phase diagrams of colloidal liquid crystals from a microscopic theory is technically involved and computationally expensive, as density profiles $\rho(z, \theta, \varphi)$ are to be determined for a given wall potential $V(z, \theta, \phi)$, with $z$ the distance from the wall and $\theta$ and $\varphi$ the polar and azimuthal angle of the orientation of the rod. Building on earlier studies [22,23], only recently a study appeared of the surface properties of a hard-spherocylinder fluid in contact with a model substrate, composed of a "penetrable" wall and a short-ranged repulsive or attractive tail [17]. Several wetting transitions and a transition from homeotropic to planar anchoring were observed at different strengths of the wall potential. This can be understood qualitatively, since the "penetrable" wall with the attractive tail favors homeotropic alignment of the nematic director, and for the strong repulsive tail it resembles the hard wall such that the stable director changes to planar. However, the complexity of the model prohibits to explore the full parameter space.

In order to be able to map a complete surface phase diagram we sacrifice the full rotational symmetry of the rods and consider the simpler Zwanzig model, with a restricted number of allowed orientations [24]. This model exhibits a strong first-order IN transition in the bulk [24], and the orientation of the nematic director parallel to the IN interface was found to be thermodynamically favorable [25], similar to continuous rods. In contact with a hard wall the WI interface is completely wet by the $\mathrm{N}$ phase upon approach of the IN coexistence, whereas the WN interface remains partially wet [25], i.e. in such a geometry the model also shows behavior similar to continuous rods. Although some limitations of the Zwanzig model are known $[26,27]$, we adopt it for the present study for reasons of its numerical simplicity. We explore the complete surface phase behavior of a Zwanzig hard-rod fluid in contact with a substrate which consists of a hard wall and a short-ranged attractive or repulsive tail. In contrast with the "penetrable" wall it puts the infinite potential barrier at the origin regardless the orientation of the rod.

We consider an inhomogeneous fluid of rectangular hard rods of length $L$ and diameter $D$ $(L \gg D)$ in a macroscopic volume $V$ at inverse temperature $\beta$ and chemical potential $\mu$. The rod orientations are restricted to the three mutually perpendicular directions $\hat{n}_{i}$ representing $\hat{x}, \hat{y}, \hat{z}$ for $i=1,2,3$, respectively. The position $\mathbf{r}$ of the center of mass of a rod is continuous. The grand potential functional of the fluid in an external potential $V_{i}(\mathbf{r})$ with the one-particle distribution functions $\rho_{i}(\mathbf{r})$ can be written, within the second virial approximation, as [25]

$\beta \Omega[\rho]=\sum_{i=1}^{3} \int d \mathbf{r} \rho_{i}(\mathbf{r})\left(\ln \left[\rho_{i}(\mathbf{r}) \nu\right]-1-\beta \mu+\beta V_{i}(\mathbf{r})\right)-\frac{1}{2} \sum_{i, j=1}^{3} \int d \mathbf{r} d \mathbf{r}^{\prime} f_{i j}\left(\mathbf{r} ; \mathbf{r}^{\prime}\right) \rho_{i}(\mathbf{r}) \rho_{j}\left(\mathbf{r}^{\prime}\right),(1)$

with $\nu=L^{2} D$, and $f_{i j}\left(\mathbf{r} ; \mathbf{r}^{\prime}\right)=-1,(0)$ the Mayer function of two (non-)overlapping hard rods with orientations $\hat{n}_{i}$ and $\hat{n}_{j}$ and center-of-mass coordinates $\mathbf{r}$ and $\mathbf{r}^{\prime}$. Qualitatively, the second virial functional predicts the bulk IN transition and the adsorption and wetting properties near a planar hard wall in agreement with simulations of freely rotating rods [25].

The external potential $V_{i}(\boldsymbol{r})$ consists of a single planar hard wall in the plane $z=0$ (normal $\hat{z})$ and a generic soft "tail" $\propto \exp (-\kappa z)$ acting on each rod segment, and is written as

$$
\beta V_{i}(\mathbf{r})= \begin{cases}\beta A \exp [-\kappa z] & z>0, i=1,2 \\ \beta A F \exp [-\kappa z] & z>\frac{L}{2}, i=3 \\ \infty & \text { otherwise }\end{cases}
$$


where the form factor $F=\sinh (\kappa L / 2) /(\kappa L / 2)>1$ arises from integration over the contour of the rod. For $A>0$ such a potential could, under certain circumstances, be caused by a polymer coating, with $A$ a measure for the planar density and $\kappa^{-1}$ for the radius of gyration [28]. In the present study we avoid discussions on the microscopic origin of $V_{i}(\mathbf{r})$, and explore the generic properties of the surface phase diagram as a function of the "contact" potential $A$ and the decay constant $\kappa$. Our primary interest is in the parameter regime $\beta A \leq 10$, since larger values of $\beta A$ correspond essentially to a shift of the hard wall along the $z$-axis.

Throughout we assume translational invariance in the $x y$ plane, such that the dimensionless densities $c_{i}(z)=\rho_{i}(\mathbf{r}) \nu$ only depend on the distance from the substrate $z$. The Euler-Lagrange equations $\delta \Omega[\{\rho\}] / \delta \rho_{i}(\mathbf{r})=0$ can then be written as [25]

$$
\begin{gathered}
\beta \mu=\ln c_{1}(z)+\beta V_{1}(z)+2 c_{2}(z)+2 \bar{c}_{3}(z), \\
\beta \mu=\ln c_{2}(z)+\beta V_{2}(z)+2 c_{1}(z)+2 \bar{c}_{3}(z), \\
\beta \mu=\ln c_{3}(z)+\beta V_{3}(z)+2 \bar{c}_{1}(z)+2 \bar{c}_{2}(z),
\end{gathered}
$$

with the averaged densities $\bar{c}_{i}(z)=(1 / L) \int_{-L / 2}^{L / 2} d z^{\prime} c_{i}\left(z+z^{\prime}\right)$. We solve Eqs. (3) iteratively for a given $A$ and $\kappa$ at a fixed chemical potential $\mu$. In all numerical calculations we use an equidistant $z$-grid of 40 points per $L$. Convergence is assumed when the relative difference between the results of the subsequent iterations is smaller then $10^{-10}$ for all values of $z$ in the grid. Such accuracy is required to avoid dependencies of the results of the calculations on the initial guesses. Additional checks, performed with 80 points per $L$, yield virtually identical results. Once determined, the equilibrium profiles can be inserted into the functional to obtain the equilibrium value of the grand potential

$$
\beta \Omega=\frac{\mathcal{A}}{2 \nu} \sum_{i} \int d z c_{i}(z)\left(\ln \left[c_{i}(z)\right]-2-\beta \mu+\beta V_{i}(z)\right) .
$$

Note that $\Omega=-p V$ for a bulk system in a volume $V$, with $p=p(\mu, T)$ the pressure. In the presence of a planar surface or interface of area $\mathcal{A}$ we have $\Omega=-p V+\gamma \mathcal{A}$ with $\gamma=$ $\gamma(\mu, T)$ the surface or interface tension. The results of the calculations can be conveniently represented in terms of the total density $c(z)=\sum_{i=1}^{3} c_{i}(z)$, the nematic order parameter $s(z)=\left(c_{3}(z)-\frac{1}{2}\left(c_{1}(z)+c_{2}(z)\right)\right) / c(z)$, and the biaxiality $\Delta(z)=\left(c_{1}(z)-c_{2}(z)\right) / c(z)$. The (dimensionless) adsorption near a planar substrate is defined as $\Gamma(\mu)=\int_{0}^{\infty} d z\left(c(z)-c_{b}\right) / L$, where $c_{b}=c_{b}(\mu)$ is the bulk density far from the substrate. In Fig. 1(a,b,c) we show $\mu-A$ surface phase diagrams for several ranges of the wall potential $\kappa L=6$ (a), $\kappa L=2(\mathrm{~b})$, and $\kappa L=1$ (c). The phase diagrams are very rich, therefore we discuss separately their parts which correspond to the low $\left(\mu \leq \mu_{I N}\right)$ and high $\left(\mu>\mu_{I N}\right)$ values of the bulk chemical potential.

For $\mu \leq \mu_{I N}$ all three phase diagrams of Fig. 1(a,b,c) exhibit a uniaxial-to-biaxial (UB) surface transition at $\mu=\mu_{U B}(A)<\mu_{I N}$ for small enough $A<A_{U B}^{c}$. This implies that only sufficiently repulsive walls do not show the UB transition, such that $\Delta(z) \equiv 0$ even upon approach of $I N$ coexistence. The critical value satisfies $\beta A_{U B}^{c}>0.5$ in all cases, and increases with $\kappa L$ (Fig. 1(d)). The $U B$ transition is found to be continuous for all investigated combinations of $\kappa L$ and $\beta A$. This is consistent with the findings of Ref. [25], where the case $\beta A=0$ (hard-wall) was considered.

For all studied $\kappa L$ we only recover the hard-wall complete wetting phenomenon [25]) for sufficiently weak repulsions $A<A_{w}$, whereas strong repulsions $A>A_{w}$ give rise to partial 

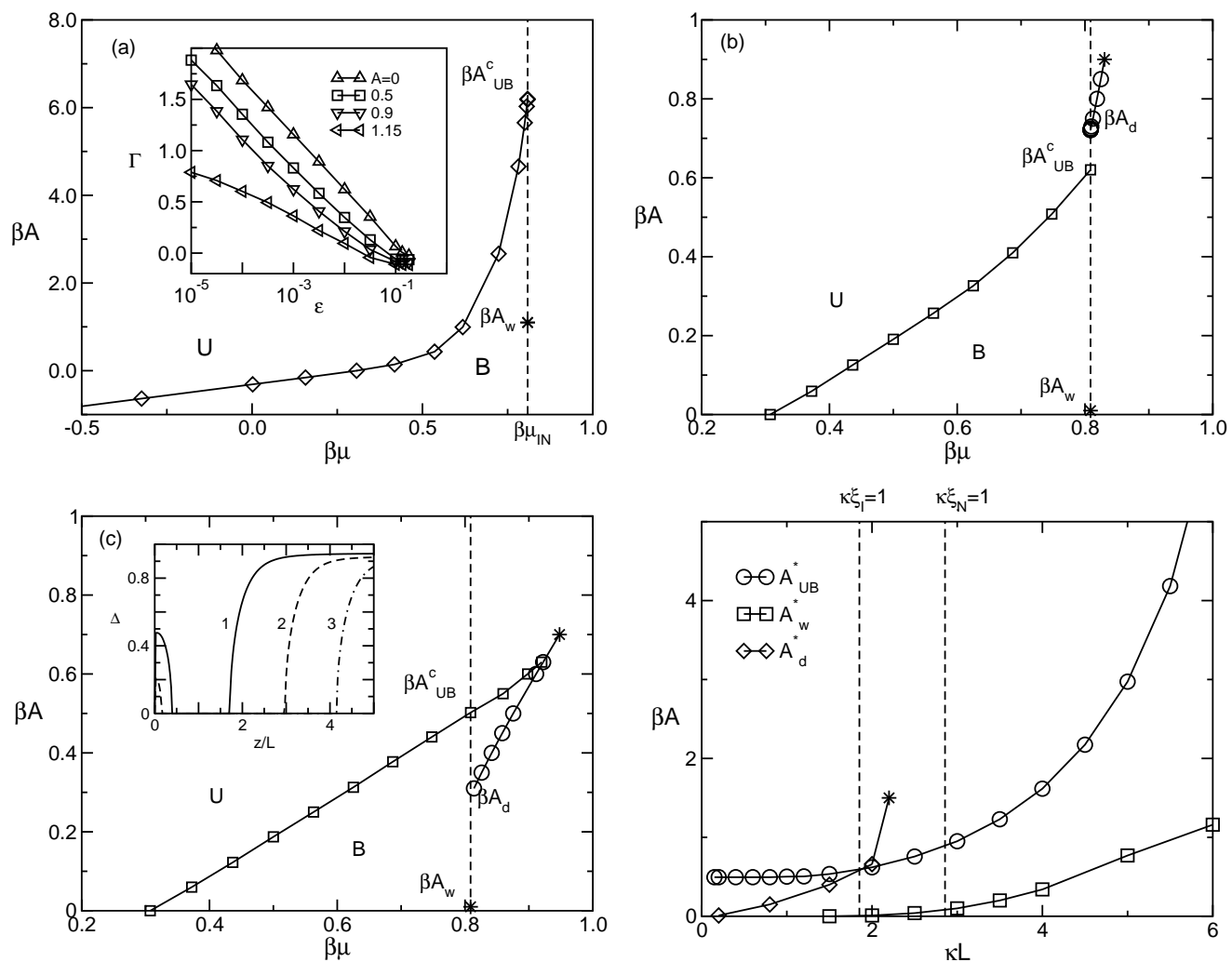

Fig. 1 - (a,b,c) Surface phase diagrams of the Zwanzig hard-rod fluid in contact with a soft wall in terms of the contact potential $\beta A$ and chemical potential $\beta \mu$ for different range of the wall potential $\kappa L=6(\mathrm{a}), \kappa L=2(\mathrm{~b})$, and $\kappa L=1(\mathrm{c})$. In all three cases we distinguish a uniaxial $(U)$ and biaxial $(B)$ phase for $\mu<\mu_{I N}$, and a wetting transition $(*)$ at $\mu=\mu_{I N}$ that separates complete wetting of the wall by a nematic film at $A<A_{w}$ from partial wetting $A>A_{w}$. In (b) and (c) we also see a predrying line at $\mu>\mu_{I N}$, and a drying transition at $\beta \mu=\beta \mu_{I N}$, that separates complete drying by an isotropic film at $A>A_{d}$ from partial drying $A<A_{d}$. The inset in (a) illustrates the logarithmic divergence of the adsorption $\Gamma$ with undersaturation $\epsilon=1-c_{b} / c_{I}$ for $A<A_{w}$, and that in (c) shows several biaxiality profiles $\Delta(z)$ for $A=0.55>A_{U B}^{c}$ for several values of the bulk chemical potential $\mu_{1,2,3}>\mu_{I N}$. (d) Generic surface phase diagram for a fluid of Zwanzig hard rods at IN coexistence in contact with the "soft" wall. Solid lines correspond to the wetting $(\square)$, drying $(\diamond)$, and the UB (०) transitions; the end point on the line of drying transitions is indicated by $(*)$. The dashed lines indicate $\kappa \xi_{I, N}=1$, with the bulk correlation lengths $\xi_{I} / L=0.54$ and $\xi_{N} / L=0.35$ of the bulk I and $\mathrm{N}$ phase, respectively.

wetting. This is illustrated in the inset of Fig.1(a), where the adsorption $\Gamma$ diverges logarithmically with the undersaturation $\epsilon=1-c_{b} / c_{I} \rightarrow 0$ for $A<A_{w} \simeq 1.1$, and remains finite for $A>A_{w}$. Here $c_{I}$ is the coexisting isotropic bulk density. This implies that a wetting transition takes place at $A=A_{w}$. Interestingly, the numerical value $A_{w} \simeq 10^{-2}$ is seen to be very small for longer-ranged potentials, $\kappa L \leq 2$, i.e. extremely weak but sufficiently long-ranged repulsions strongly reduce the complete-wetting regime. Shorter-ranged substrate potentials exhibit a larger complete wetting regime, e.g. $A_{w}=\simeq 1.1$ when $\kappa L=6$ (see also Fig凹(d)). The existence of a wetting transition at $A=A_{w}$ follows also from comparisons of 

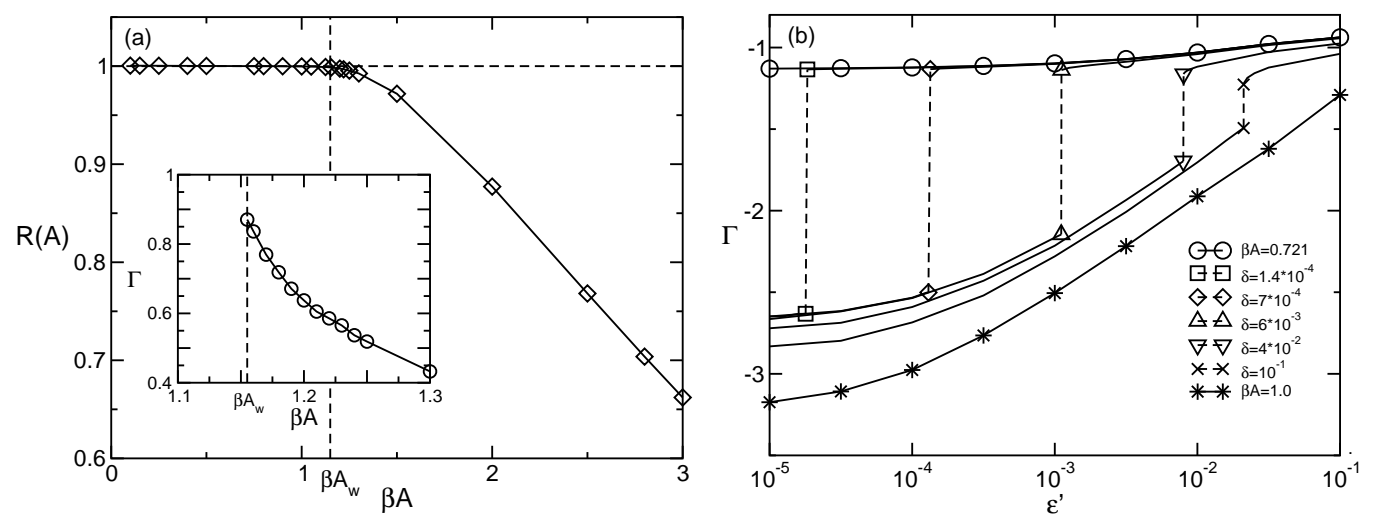

Fig. 2 - (a) The relative surface tension difference $R(A)$ at the $W I$ and $W N$ interfaces for a wall potential with a decay given by $\kappa L=6$. The vertical dashed line $\beta A_{w}=1.15$ indicates the repulsion amplitude at which the wetting transition takes place. The inset shows the excess adsorption $\Gamma(A)$ at coexistence. (b) Excess desorption $\Gamma\left(\epsilon^{\prime}\right)$ at the interface between the $N$ phase and the soft wall $(\kappa L=$ 2 ) as a function of the oversaturation $\epsilon^{\prime}=c_{b} / c_{N}-1$ for several different values of $\beta A=0.721(1+\delta)$. The vertical dashed lines connect coexisting points at the predrying line.

the corresponding interfacial energies. For convenience, we define the relative surface tension difference

$$
R(A)=\frac{\gamma_{W I}(A)-\gamma_{W N}(A)}{\gamma_{I N}}
$$

with $\gamma_{W I}(A)$ and $\gamma_{W N}(A)$ the tensions of the $W I$ and $W N$ interface, respectively, both at $\mu=\mu_{I N}$, and $\beta \gamma_{I N} L D=(2.8027 \pm 0.0001) \times 10^{-2}$ the surface tension of the free planar $I N$ interface [25]. In Fig. 2(a) we show $R(A)$ for $\kappa L=6$. It can be seen that $R(A) \equiv 1$ (with relative accuracy of $10^{-3}$ ) if $\beta A<\beta A_{w}=1.15$, and $R(A)<1$ if $A>A_{w}$. This is a clear thermodynamic evidence for a transition from partial to complete wetting at $A=A_{w}$.

The study of the order of the wetting transition represents significant numerical difficulties. Our results suggest that it is likely a first-order wetting transition, because (i) $R(A)$ appears to approach $R\left(A_{w}\right)=1$ with a finite slope when $A \downarrow A_{w}$, (ii) metastable nematic films of finite thickness can easily be generated at $\mu=\mu_{I N}$ for $A<A_{w}$, and (iii) the adsorption $\Gamma\left(\mu_{I N}\right)$ at saturation appears to show a discontinuous jump from a finite value for $A>A_{w}$ to an infinite value for $A<A_{w}$ (see insets Fig. 1(a) and 2(a)). One expects, for reasons of continuity, that a first-order wetting transition is accompanied by a first-order prewetting transition, from a finite thin to a finite thick film off coexistence. Unfortunately, we have not been able to find this prewetting transition, despite considerable efforts. We speculate that its critical point is too close to the wetting transition to be detected.

We now consider the $\mu>\mu_{I N}$ part of the phase diagrams, where the $N$ bulk phase has director $\hat{x}$, i.e. planar. We have checked that a homeotropic orientation of the director is metastable in all the cases studied. Intuitively, one expects a low-density isotropic film close to a highly repulsive substrate $(\beta A \gg 1)$, which may but need not become of macroscopic thickness when $\mu \downarrow \mu_{I N}$. For $\beta A=0$ it is known that $\Gamma<0$ but finite [25], a situation that we refer to as partial drying. Our numerical results for $\kappa L=6$ show that $\Gamma$ remains finite when $\mu \downarrow \mu_{I N}$ for any $\beta A<10$. For $\beta A \geq 10$ the density of rods close to the hard wall $(z=0)$ is so small that its position can be shifted to some $z=z_{0}>0$ without affecting the profiles 
$c_{i}(z)$ significantly, while the effective amplitude is reduced from $A$ to $A \exp \left(-\kappa z_{0}\right) \leq 10$. We can conclude that the soft potential with $\kappa L=6$ is so short-ranged that the partial drying is obtained for any value of $\beta A$. Fig.1(a) is therefore featureless for $\mu \geq \mu_{I N}$.

The situation is more interesting for the longer-ranged cases $\kappa L=2,1$, where we do find a drying transition at $\beta A_{d}=0.72,0.31$ in Fig. 团 b), (c), respectively. When $\mu \downarrow \mu_{I N}$ partial drying is observed for $A<A_{d}$, and complete drying by an isotropic film for $A>A_{d}$. In Fig. 2(b) we show the excess adsorption $\Gamma\left(\epsilon^{\prime}\right)$ as a function of oversaturation $\epsilon^{\prime}=c_{b} / c_{N}-1$ for various amplitudes of the wall potential with $\kappa L=2$. At low values of the contact potential $\left(A \leq A_{d}\right)$ the desorption remains almost constant upon approach of the $I N$ coexistence. Calculations show that $R(A)>-1$ in this regime, corresponding to partial drying. The behavior changes qualitatively for $0.72<\beta A<0.95$, where $\Gamma\left(\epsilon^{\prime}\right)$ exhibits a discontinuity at some $\epsilon^{\prime}>0$, which we associate with predrying. For $\beta A \simeq 0.95$ the jump of the desorption takes place at $\epsilon^{\prime}=0.05$, and its magnitude is (vanishingly) small, i.e. this is the critical predrying point indicated by $(*)$ in Fig. I(b) and (c). The jump of the desorption at the predrying line increases upon decreasing $\beta A$ towards $\beta A_{d}$, and its location shifts to smaller $\epsilon^{\prime}$, until it diverges at the drying transition at $\beta A=\beta A_{d}$ at $\epsilon^{\prime} \rightarrow 0$. For $\beta A \geq 1.0$, i.e. above the critical predrying amplitude, the desorption grows continuously upon approach of coexistence. Studies of the surface tensions at coexistence show that $R(A)=-1.000 \pm 0.001$ for all $A>A_{d}$, consistent with a complete drying of the $W N$ interface by an intervening isotropic film. However, the profiles (not shown) do not exhibit a clear bulk film even at $\epsilon^{\prime}=10^{-5}$, which implies that the asymptotic limit $\epsilon^{\prime} \rightarrow 0$ has not been reached yet such that the regime of logarithmic growth of $-\Gamma$ with $\epsilon^{\prime}$ cannot properly be identified in Fig. 2(b). Interestingly, for $\kappa L \leq 1.5$ we find that $A_{d}<A_{U B}^{c}$, such that for $A>A_{d}$ the (thick) isotropic film that grows in between the wall and the nematic bulk phase can exhibit a UB transition. In other words, the UB transition at $\mu<\mu_{I N}$ extends continuosly to the thick isotropic films at $\mu>\mu_{I N}$, as illustrated for $\kappa L=1$ in Fig. 1(c). Here the line of $U B$ transitions terminates at $\beta A_{U B}^{*}=0.63$, where it crosses the predrying line. We note, finally, that no new physics is to be expected for larger $A$ in Fig प(b) and (c), since the regime of approximate scaling of the density profiles (associated with a shift of the hard wall and a renormalization of the contact potential) at large $A$ is observed already for $\beta A \geq 1.5$.

All our findings are summarized in Fig. [1(d), which represents the generic surface phase diagram in the $\beta A-\kappa L$ coordinates for a fluid of Zwanzig hard rods at $I N$ coexistence $\left(\mu=\mu_{I N}\right)$ in contact with the "soft" wall. We distinguish lines of the wetting $(\square)$ and drying $(\diamond)$ transitions. The $U B$ transition (o) at the $W I$ interface occurs for all $\kappa L$. It also takes place at the $W N$ interface in the thick isotropic wetting film, i.e. below the intersection of the lines of the drying and the $U B$ transitions. Note that the growth of the wetting film is determined by the correlation length $\xi_{I}\left(\xi_{N}\right)$ of the coexisting bulk isotropic and nematic phase, and no longer by $\kappa^{-1}$, when $\kappa^{-1}<\xi_{I}\left(\xi_{N}\right)$. However, this crossover does not introduce any additional structure to the surface phase diagram. For large $\kappa L$ the $U B$ line shows an exponential divergence $\beta A \sim \exp [\kappa L]$, associated with the renormalization of the contact potential in the scaling regime $\beta A=\beta A_{0} \exp \left[\kappa L z_{0} / L\right]$. The line of the wetting transitions is seen to be linear with $\kappa L>4$. This is associated with the fact that for the steep potentials density variations within the decay length of the potential are rather small, and can be neglected. Therefore, its contribution to the grand potential is proportional to $(\beta A / L) c(0) \int d z \exp [-\kappa z]=(\beta A / \kappa L) c(0)$. Since the wetting transition for large $\kappa L$ occurs at similar values of the surface free energy, $\left(\beta A_{w} / \kappa L\right) c(0)=$ const, and hence $\beta A_{w} \sim \kappa L$.

We conclude that the surface phase behaviour of hard rods with soft walls is extremely rich, at least on the basis of the present analysis within the Zwanzig model. We find that complete wetting of the WI interface by a nematic film, as found for a planar hard wall, 
is very sensitive to weak but long-ranged wall repulsions, and that strongly repulsive walls show complete drying of the WN interface by an intervening isotropic film if the range of the potential is long enough. It is of interest to extend the present study to other related systems, e.g. freely rotating rods or platelets. Another related interesting question concerns the required nature of the surface potential that can lead to a stable anchoring transition in a colloidal-rod fluid [23]. Work along these lines is in progress, and hopefully stimulates the study of colloidal analogues of liquid crystals near interfaces.

\section{$* * *$}

It is a pleasure to thank M. Dijkstra, R. Evans and C. Likos for stimulating discussions. This work is part of the research program of the "Stichting voor Fundamenteel Onderzoek der Materie (FOM)", which is financially supported by the "Nederlandse organisatie voor Wetenschappelijk Onderzoek (NWO)".

\section{REFERENCES}

[1] L. Onsager, Ann. N.Y. Acad. Sci. 51, 627 (1949).

[2] G.J. Vroege and H.N.W. Lekkerkerker, Rep. Prog. Phys. 55, 1241 (1992).

[3] P. Bolhuis and D. Frenkel, J. Chem. Phys. 106, 666 (1997).

[4] A. M. Somoza and P. Tarazona, Phys. Rev. Lett. 61, 2566 (1988).

[5] A. Poniewierski and R. Holyst, Phys. Rev. Lett. 61, 2461 (1988).

[6] L. Mederos and D. E. Sullivan, Phys. Rev. A 39, 854 (1989).

[7] Z.Y. Chen and J. Noolandi, Phys. Rev. A 45, 2389 (1992).

[8] M. Allen, J. Chem. Phys. 112, 5447 (2000).

[9] K. Shundyak and R. van Roij, J. Phys.: Condens. Matter 13, 4789 (2001).

[10] K. Shundyak and R. van Roij, Phys. Rev. Lett. 88, 205501 (2002).

[11] Z.Y. Chen, Phys. Rev. E 47, 3765 (1993).

[12] K. Shundyak and R. van Roij, Phys. Rev. E 68, 061703 (2003).

[13] S. Wolfsheimer, C. Tanase, K. Shundyak, R. van Roij and T. Schilling, submitted.

[14] A. Poniewierski and R. Holyst, Phys. Rev. A 38, 3721 (1988).

[15] M. Dijkstra, R. van Roij, and R. Evans, Phys. Rev. E 63, 051703 (2001).

[16] M.P. Allen, Mol. Phys. 96, 1391 (1999).

[17] D. de las Heras, L. Menderos and E. Velasco, Phys. Rev. E 68, 031709 (2003).

[18] P. Sheng, Phys. Rev. Lett. 37, 1059 (1976); Phys. Rev. A 26, 1610 (1982).

[19] T.J. Sluckin and A. Poniewierski, Phys. Rev. Lett. 55, 2907 (1985).

[20] B. Jérôme, Rep. Prog. Phys. 54, 391 (1991), and references therein.

[21] A.K. Sen and D.E. Sullivan, Phys. Rev. A 35, 1391 (1987).

[22] M.M. Telo da Gama, Mol. Phys. 52, 611 (1984).

[23] I. Rodríguez-Ponce, J.M. Romero-Enrique, E. Velasco, L. Mederos, and L.F. Rull, Phys. Rev. Lett. 82, 2697 (1999).

[24] R. Zwanzig, J. Chem. Phys. 39, 1714 (1963).

[25] R. van Roij, M. Dijkstra and R. Evans, Europhys. Lett. 49, 350 (2000); J. Chem. Phys. 113, 4799 (2000).

[26] K. Shundyak and R. van Roij, Phys. Rev. E 69, 041703 (2004).

[27] J.P. Straley, J. Chem. Phys. 57, 3694 (1972).

[28] G.J. Fleer et al., Polymers at Interfaces; Chapman and Hall: London, 1993. 